\title{
THE FAR-ULTRAVIOLET BACKGROUND
}

\author{
PETER JAKOBSEN \\ Astrophysics Division \\ Space Science Department of ESA \\ ESTEC, 2200 AG Noordwijk \\ The Netherlands
}

\section{Introduction}

Extensive reviews of the diffuse background at ultraviolet $(\lambda 1000-2500 \AA)$ wavelengths have been given by Davidsen, Bowyer \& Lampton (1974), Paresce \& Jakobsen (1980), and most recently by Bowyer (1992) and Henry (1992). Since many astronomical sources emit radiation with photon energies in the range $h \nu \simeq 10-20 \mathrm{eV}$ through various thermal and non-thermal emission processes, the diffuse UV background is of relevance for a range of topics. These include the study of the properties of interplanetary and interstellar dust grains, thermal line emission from the interstellar and intergalactic gas, the integrated light of galaxies, and radiative decay of exotic cosmological particles.

On the observational side, there is today good evidence that the diffuse UV background is largely dominated by dust scattering and other interstellar emission processes occurring within the Milky Way. The intensity of any quasi-isotropic and therefore possibly extragalactic component to the background in the $\lambda 1300-2000 \AA$ range is presently only known to within a factor of $\sim 2-3$, but is approximately $I_{\lambda} \approx 100$ photons s${ }^{-1} \mathrm{~cm}^{-2} \mathbf{s r}^{-1} \AA^{-1}$ (Martin \& Bowyer 1990, Martin, Hurwitz \& Bowyer 1990, Hurwitz, Bowyer \& Martin 1991).

Three extragalactic sources of UV radiation that could conceivably produce a diffuse background flux of this intensity: i) diffuse thermal emission from the intergalactic medium; ii) the integrated UV light of galaxies and quasars; and iii) radiative decay of massive neutrinos. This paper briefly summarizes what is currently known about the first two of these emission 
sources. For a discussion of the latter, more speculative, source, the reader is referred to the paper presented by Dr. Sciama at this symposium.

\section{Diffuse UV Emission from the Intergalactic Medium}

One of the original motivations behind the first attempts to measure the diffuse background radiation at far-ultraviolet wavelengths was the hope that such observations might reveal the existence of a dense $(\Omega \approx 1)$, "lukewarm" $\left(10^{3} \mathrm{~K}<T<10^{6} \mathrm{~K}\right)$ intergalactic medium (IGM) through the redshift-smeared HI Ly $\alpha$ and HeII $\lambda 304$ line emission that such a medium would emit (cf. Kurt \& Sunyaev 1967, Davidsen, Bowyer \& Lampton 1974, Paresce \& Jakobsen 1980).

Since then, a wealth of information about the nature, composition and physical properties of intergalactic gas has been obtained through the study of quasar absorption lines. Thanks to the extensive surveys of the discrete absorption lines that appear in quasar spectra, we today not only have a reasonably accurate measure of how much matter intergalactic space contains, but also a good picture of the ionization processes at work in this gas. Armed with this new information, it is possible to show that diffuse thermal emission from intergalactic gas is almost certainly not a significant contributor to the diffuse far-UV background. In the following, the main arguments leading to this conclusion are briefly outlined. More complete discussions can be found in Martin, Hurwitz \& Bowyer (1991) and Jakobsen (1994).

\subsection{REDSHIFTED LY $\alpha$ AND HEII $\lambda 304 \AA$ LINE EMISSION}

From the study of absorption lines in quasar spectra, it is known that intergalactic space contains significant amounts of baryonic matter, in the form of an abundant population of hydrogen "Lyman forest" clouds (Sargent et al. 1980, Murdoch et al. 1986, Sargent 1988, Hunstead 1988), and most likely also in the form of a diffuse "intercloud" IGM (Jakobsen et al. 1994).

Both the so-called Lyman forest clouds and the ambient IGM are believed to be kept highly photoionized by a metagalactic flux of ionizing radiation, presumably arising from the ionizing output from quasars and young galaxies. One key piece of evidence for this is found in the so-called "proximity effect" (Carswell et al. 1982; Murdoch et al. 1986; Bajtlik, Duncan \& Ostriker 1988; Lu, Wolfe \& Turnshek 1991, Bechtold 1994), i.e. the statistical underabundance displayed by the forest systems as the emission redshift of a given quasar is approached. This effect is interpreted as being caused by the radiation field of the background quasar enhancing the total ionizing flux above the metagalactic level. Since the quasar flux can be estimated from the magnitude and spectrum of the quasar, an estimate of the 
background metagalactic ionizing background intensity can be derived from the measured contrast of the effect. Through this technique one infers that the intergalactic ionizing background in the redshift range $1.7 \lessgtr z \lesssim 3.8$ has an intensity at the Lyman limit of order $I_{\nu_{H}} \approx 10^{-21} \mathrm{erg} \mathrm{s}^{-1} \mathrm{~cm}^{-2} \mathrm{sr}^{-1} \mathrm{~Hz}^{-1}$. At lower redshifts, the proximity effect is less pronounced and the inferred extragalactic ionizing background is approximately two orders of magnitude lower (Kulkarni \& Fall 1993); and consistent with limits on the local extragalactic ionizing flux derived from $\mathrm{H} \alpha$ observations of high-latitude and extragalactic HI clouds (Reynolds et al. 1986; Songaila, Bryant \& Cowie 1989; Kutyrev \& Reynolds 1989)

If ionized, both the Lyman forest clouds and the ambient IGM will emit Ly $\alpha \lambda 1216 \AA$ and HeII $\lambda 304$ line radiation through the process of recombination. A firm upper limit on the diffuse background contribution due to this redshifted recombination emission can be placed from the following simple argument. Since a photoionized gas effectively acts as a simple photon down-converter (emitting for example $\sim 0.7$ Ly $\alpha$ photons per photoionization event), it follows that the intensity of the resulting recombination emission can never exceed that of the ionizing input flux. Therefore the background at observed wavelength $\lambda$ due to redshift-smeared recombination radiation in a line at wavelength $\lambda_{l}$ must obey

$$
I_{\lambda}(\lambda) \ll\left[\frac{I_{\nu_{H}}(z)}{2 \pi \hbar \lambda_{H}(1+z)^{4}}\right]
$$

where the expression on the right is merely the ionizing background intensity $I_{\nu_{H}}(z)$ at the Lyman limit, $\lambda_{H}$, converted to photon units and redshifted to $z=0$ from the appropriate redshift, $z=\lambda / \lambda_{l}-1$.

In the case of the Ly $\alpha$ line, emission from redshifts $0 \lesssim z \lessgtr 0.6$ will give rise to diffuse background radiation at wavelengths $\lambda \simeq 1200-2000 \AA$. The ionizing flux at these redshifts of $I_{\nu_{H}} \approx 10^{-23} \mathrm{erg} \mathrm{s}^{-1} \mathrm{~cm}^{-2} \mathrm{sr}^{-1} \mathrm{~Hz}^{-1}$ yields per eq.(1) an upper limit on the observable background of $I_{\lambda} \ll 2$ photon$\mathrm{s} \mathrm{s}^{-1} \mathrm{~cm}^{-2} \mathrm{sr}^{-1} \AA^{-1}$. Consequently, redshifted intergalactic recombination emission in Ly $\alpha$ can at most contribute a few percent of the nominal observed extragalactic UV flux.

This simple, but powerful, argument applies equally well to the case of redshifted HeII $\lambda 304 \AA$ recombination from higher redshifts $3 \lesssim z \lesssim 5$. Since the spectrum of the ionizing background is likely to decrease with decreasing wavelength, the ionizing background at the $\lambda_{H e I I}=228 \AA$ photoionization threshold of singly ionized helium is almost certainly not higher than that at $\lambda_{H}=912 \AA$. The nominal ionizing background at $z \gtrsim 3$ of $I_{\nu_{H}} \approx 10^{-21} \mathrm{ergs} \mathrm{s}^{-1} \mathrm{~cm}^{-2} \mathrm{sr}^{-1} \mathrm{~Hz}^{-1}$ corresponds in this case to a redshifted background of $I_{\lambda} \ll 1$ photons $\mathbf{s}^{-1} \mathrm{~cm}^{-2} \mathbf{s r}^{-1} \AA^{-1}$. Hence, it is safe to con- 
clude that redshifted recombination emission in HeII $\lambda 304 \AA$ also occurs at far too low an intensity to be of any practical importance.

The alternative possibility of collisionally excited Ly $\alpha$ emission - as would arise in a shock-heated IGM - can also be ruled out at a high level of confidence. In this case the intensity of collisionally excited Ly $\alpha$ emission constrained to unobservably low levels by the firm upper limit on the total amount of neutral hydrogen present in intergalactic space as determined from quasar absorption line statistics and the Gunn-Peterson test (see Jakobsen 1994 for details).

The equivalent argument does not apply in the case of collisionally excited HeII $\lambda 304 \AA$ emission, since the sole detection of strong HeII GunnPeterson absorption in a high redshift quasar obtained so far only provides a lower limit on the amount of intergalactic HeII present at $z \sim 3$ (Jakobsen et al. 1994). If the IGM is sufficiently dense $(\Omega \sim 1)$ and collisionally ionized, it could in principle emit strongly in HeII $\lambda 304 \AA$. However, even so, the resulting background flux would still not be observable because the universe is optically thick to HeII $\lambda 304 \AA$ radiation at large redshift.

\subsection{THE LYMAN CONTINUUM OPACITY OF THE UNIVERSE}

Any emission originating at high redshift at wavelengths below the Lyman limit is subject to photoelectric absorption by neutral hydrogen encountered along its path. Although it has been known for some time that the classical Gunn-Peterson test demonstrates that the Lyman continuum opacity of any smoothly distributed IGM is negligible, it has only recently been fully appreciated that the statistics of quasar absorption lines imply the that the accumulated absorption out to moderate and high redshift from the clumped component is quite substantial.

Detailed discussions of this topic have been given by Møller \& Jakobsen (1990) and Zuo \& Phinney (1993), who show that the cumulative absorption is dominated by the densest so-called "Lyman limit" types of quasar absorption systems (Tytler 1982; Bechtold et al. 1984; Lanzetta 1988; Sargent, Steidel \& Boksenberg 1989; Bahcall et al. 1993) In the particular case of HeII $\lambda 304 \AA$ emission stemming from redshifts $z \gtrless 3$, such radiation will be attenuated by approximately two orders of magnitude of accumulated photoelectric absorption out to such high redshift (cf. Jakobsen 1994, Figure 3).

\section{The Integrated UV Light of Galaxies and Quasars}

The brightness of the extragalactic sky at visible and infrared wavelengths carries important information on the history of star formation and galaxy evolution throughout the age of the Universe. As first emphasized by Tins- 
ley (1973), the diffuse background at the shorter UV wavelengths is also an important element of the modern day version of "Olbers' paradox" - but with several key differences with respect to the situation in the adjacent spectral regions.

For one, the observed integrated far-UV spectra of the different classes of galaxies are not particularly well modelled or understood at present. This is especially true of the UV emission from ellipticals and the bulges of spirals, which is believed reflect complex stages of late stellar evolution (cf. Burnstein et al. 1988; King et al. 1993 and references therein). However, the global galaxy luminosity function at UV wavelengths is almost entirely dominated by the emission from massive $\mathrm{O}$ and $\mathrm{B}$ stars contained in the star-forming regions of spirals and irregulars. Consequently, in the UV late type galaxies account for close to $90 \%$ of the local galaxy luminosity density and therefore dominate the integrated galaxy background (cf. Armand \& Milliard 1994 for a recent discussion).

Another important difference with respect to the case at visible and longer wavelengths is that the integrated UV light of galaxies is accumulated over a relatively modest cosmological pathlength. Late type galaxies are generally surrounded by large halos of neutral hydrogen which permit little or no radiation to escape below the Lyman limit at $\lambda \leq 912 \AA$. At an observed wavelength of $\lambda 1500 \AA$, the Lyman limit is reached at $z=0.64$, corresponding to a look-back time of $\Delta t \simeq 3 h^{-1}$ Gyr. This modest lookback time, combined with the short life times of main sequence $O B$ stars, leads to the integrated UV light of galaxies being primarily a measure of the level of on-going star formation in the relatively local Universe.

Three rather different observational and theoretical approaches have so far been employed in assessing the possible contribution of galaxies to the extragalactic UV background.

\subsection{INTEGRATION OF THEORETICAL GALAXY EVOLUTION MODELS}

Following the pioneering work of Tinsley (1972), several groups have in recent years developed very elaborate and physically self-consistent models for galaxy evolution based on stellar formation and evolution theory (e.g. Guiderdoni \& Rocca-Volmerange 1991, Bruzual \& Charlot 1993). Although the primary motivation for these models is to explain faint galaxy counts and colors observed in the visible, as first emphasized by Tinsley (1973), the predicted integrated background spectrum provides an important observational constraint on the models.

A recent discussion with emphasis on the ultraviolet has been given by Martin, Hurwitz \& Bowyer (1991), who show that the current evolution models predict a rather wide range of spectral shapes for the background. 
Nonetheless, the predicted far-UV intensities generally span the $I_{\lambda} \simeq 40-$ 240 photons $\mathrm{s}^{-1} \mathrm{~cm}^{-2} \mathrm{sr}^{-1} \AA^{-1}$ range - suggesting that the integrated UV light of galaxies should be within reach observationally.

\subsection{UV BACKGROUND FLUCTUATION MEASUREMENTS}

Martin \& Bowyer (1989) have attempted to detect the small-scale fluctuations in the far-UV background expected from the integrated light of galaxies. This technique, which was first pioneered by Schectman (1974) at visible wavelengths, consists of fitting the measured power spectrum of the UV background to that expected from galaxies calculated on the basis of observed visible light correlation functions and assumed models for galaxy spectral evolution. Based on their measurements, Martin \& Bowyer concluded that the integrated light of galaxies can at most contribute $\approx 20 \%$ of the total (galactic and extragalactic) background observed, corresponding to an intensity of $I_{\lambda} \simeq 40 \pm 13$ photonss ${ }^{-1} \mathrm{~cm}^{-2} \mathrm{sr}^{-1} \AA^{-1}$. Subsequent analysis, however, has questioned the validity of the fluctuation results due to contamination from UV starlight scattered off the IRAS cirrus (Sasseen et al. 1993).

\subsection{EXTRAPOLATION OF ULTRAVIOLET GALAXY COUNTS}

The most convincing and direct demonstration that galaxies must provide a significant contribution to the extragalactic UV background has recently been given by Armand, Milliard \& Deharveng (1994). These authors base their analysis on observed UV $(\simeq 2000 \AA)$ galaxy counts obtained with a balloon borne UV telescope. Their UV counts are complete down to a UV magnitude of $m \simeq 18.5$, which in itself yields an integrated galaxy background of $I_{\lambda} \simeq 30$ photons $\mathrm{s}^{-1} \mathrm{~cm}^{-2} \mathrm{sr}^{-1} \AA^{-1}$. Armand et al. extrapolate this resolved portion of the background to fainter magnitudes by use of theoretical galaxy evolution models. Because of the various observational and theoretical uncertainties, the total background can only be predicted with certainty to lie in the range $I_{\lambda} \simeq 40-130$ photons $\mathrm{s}^{-1} \mathrm{~cm}^{-2} \mathrm{sr}^{-1} \AA^{-1}$. Nonetheless, this flux is in good agreement with those obtained through the other less direct approaches above, and clearly demonstrates that galaxies must be a significant source of extragalactic UV background radiation.

\subsection{THE INTEGRATED UV LIGHT OF QUASARS AND AGNS}

As opposed to the situation at higher $\mathrm{X}$-ray energies, quasars and active galactic nuclei play only a marginal role in the case of the background in the UV (Martin \& Bowyer 1989; Martin et al. 1991 and references therein). The 
reason that quasars are a dominant background source in the $\mathrm{X}$-rays, but not in the adjacent $\mathrm{UV}$, can be traced to the fact that the nominal extragalactic far-UV background intensity of $I_{\lambda} \approx 100$ photons s${ }^{-1} \mathrm{~cm}^{-2} \mathrm{sr}^{-1} \AA^{-1}$ is considerably brighter in terms of energy per octave in frequency than the extragalactic background flux in the X-rays.

\section{Summary and Conclusions}

Of the various sources that have been proposed as contributors to the $d$ iffuse extragalactic background at UV wavelengths, the integrated light of galaxies is the only component that is likely to be detectable in practice. Direct galaxy counts in the UV down to a limiting magnitude of $m \simeq 18.5$ have already "resolved" the extragalactic UV background to an intensity of $I_{\lambda} \simeq 30$ photons $\mathrm{s}^{-1} \mathrm{~cm}^{-2} \mathrm{sr}^{-1} \AA^{-1}$, which extrapolated to fainter magnitudes by means of galaxy evolution models suggests that the integrated UV light of galaxies is the all-dominant source of extragalactic UV background radiation.

Thermal emission from the intergalactic medium is almost certainly an entirely negligible contributor to the UV background. The intensity of the metagalactic ionizing background at high redshifts inferred from the socalled "proximity effect" displayed by the Lyman forest absorption lines implies that the redshift-smeared backgrounds due to Ly $\alpha$ and HeII $\lambda 304 \AA$ recombination radiation from photoionized intergalactic gas are to be found at intensities far below current observational limits on the extragalactic background flux. Similarly, the results of the Gunn-Peterson test at low redshifts derived from UV quasar spectra constrain the intensity of redshifted collisionally excited Ly $\alpha$ emission from a shock-heated IGM component to an equally low intensity. Lastly, the frequency of optically thick quasar absorption systems assures that any collisionally excited HeII $\lambda 304 \AA$ emission stemming from $z \gtrsim 3$ will remain effectively hidden from view because of strong accumulated Lyman continuum absorption.

\section{References}

Armand, C, \& Milliard, B. 1994, A\&A 282,1

Armand, C, Milliard, B., \& Deharveng, J. M. 1994, A\&A 284,12

Bahcall, J. N. et al. 1993, ApJS 87,1

Bajtlik, S., Duncan, R. C., \& Ostriker, J. P. 1988, ApJ 327,570

Bechtold, J. 1994, ApJS 91,1

Bechtold, J., Green, R. F., Weymann, R. J., Schmidt, M., Estabrook, F. B., Sherman, R. D., Wahlquist, H. D., \& Heckman, T. M. 1984, ApJ 281,76

Bowyer, S. 1992, ARA\&A 29,59

Bruzual A., G. \& Charlot, S. 1993, ApJ 405,538

Burnstein, D., Bertola, F., Buson, L. M., Faber, S. M., \& Lauer, T. R. 1988, ApJ 328,440 
Carswell, R. F., Whelan, J. A. J., Smith, M. G., Boksenberg, A., \& Tytler, D. 1982, MNRAS, 198,91

Davidsen, A., Bowyer, S., \& Lampton, M. 1974, Nat 247,513

Guiderdoni, B., \& Rocca-Volmerange, B. 1991, A\&A 252,435

Henry, R. C. 1992, ARA\&A 29,89

Hunstead, R. W. 1988, in QSO Absorption Lines: Probing the Universe, ed. C. Blades, D. Turnshek, \& C. Norman, (Cambridge: Cambridge University Press), p71

Hurwitz, M., Bowyer, S., \& Martin, C. 1991, 372,167

Jakobsen, P. 1994, in Extragalactic Background Radiation, eds. M. Livio, M. Fall, D. Calzetta, \& P. Madau, Cambridge University Press (in press).

Jakobsen, P., Boksenberg, A., Deharveng, J. M., Greenfield, P., Jedrzejewski, R., \& Paresce, F. 1994, Nature 370,35

King, I. R. et al. 1993, ApJ 397,L35

Kulkarni, V. P., \& Fall, M. S. 1993, ApJ 413,L63

Kurt, V. G., \& Sunyaev, R. A. 1967, Cosmic Research, 5,496

Kutyrev, A. S., \& Reynolds, R. J. 1989, ApJ, 344,L9

Lanzetta, K. M. 1988, ApJ 332,96

Lu, L., Wolfe, A. M., \& Turnshek, D. A. 1991, ApJ 367,19

Martin, C., \& Bowyer, S. 1989, ApJ 338,667

Martin, C., \& Bowyer, S. 1990, ApJ 350,242

Martin, C., Hurwitz, M.,\& Bowyer, S. 1990, ApJ 345,220

Martin, C., Hurwitz, M.,\& Bowyer, S. 1991, ApJ 379,549

Milliard, B., Donas, J., Laget, M., Armaud, C., \& Vuillemin, A. 1992, A\&A 257,24

Murdoch, H. S., Hunstead, R. W., Pettini, M., \& Blades, J. C. 1986, ApJ, 309,19

Møller, P., \& Jakobsen, P. 1990, A\&A 228,299

Paresce, F., \& Jakobsen, P. 1980, Nature 288,119

Reynolds, R. J., Magee, K., Roesler, F. L., Scherb, F., \& Harlander, J. 1986, ApJ 309,L9

Sargent, W. L. W., 1988, in QSO Absorption Lines, Probing the Universe, ed. J. C.

Blades, D. Turnshek \& C. A. Norman (Cambridge: Cambridge University Press), p1

Sargent, W. L. W., Steidel, C. C., \& Boksenberg, A. 1989, ApJS 69,703

Sargent, W. L. W., Young, P. J., Boksenberg, A., \& Tytler, D. 1980, ApJS 42,41

Sasseen, T. P., Bowyer, S., Wu, X., \& Lampton, M. 1993, BAAS 25,822

Songaila, A., Bryant, W.,\& Cowie, L. L. 1989, 345,L71

Tinsley B. M. 1972, A\&A 20,383

Tinsley B. M. 1973, A\&A 24,89

Tytler, D. 1982, Nature 298,427

Zuo, L., \& Phinney, E. S. 1993, ApJ 418,28 